\title{
Time to Surgery and Survival in Head and Neck Cancer
}

\author{
Chandler J. Rygalski, BS ${ }^{1}$, Songzhu Zhao, MS $^{2}$, Antoine Eskander, MD, ScM ${ }^{3}$, Kevin Y. Zhan, MD ${ }^{1}$, \\ Edmund A. Mroz, PhD ${ }^{1}$, Guy Brock, $\mathrm{PhD}^{2}$, Dustin A. Silverman, $\mathrm{MD}^{1}$, Dukagjin Blakaj, $\mathrm{MD}, \mathrm{PhD}^{4}$, \\ Marcelo R. Bonomi, $\mathrm{MD}^{5}$, Ricardo L. Carrau, MD, MBA ${ }^{1}$, Matthew O. Old, MD ${ }^{1}$, James W. Rocco, MD, PhD ${ }^{1}$, \\ Nolan B. Seim, MD ${ }^{1}$, Sidharth V. Puram, MD, PhD ${ }^{6}$, and Stephen Y. Kang, MD ${ }^{1}$ \\ ${ }^{1}$ Department of Otolaryngology-Head and Neck Surgery, The Ohio State University Wexner Medical Center, Columbus, \\ $\mathrm{OH} ;{ }^{2}$ Department of Biomedical Informatics and Center for Biostatistics, The Ohio State University, Columbus, OH; \\ ${ }^{3}$ Department of Otolaryngology-Head and Neck Surgery, Sunnybrook Health Sciences Center, ON, Toronto, Canada; \\ ${ }^{4}$ Department of Radiation Oncology, The Ohio State University Wexner Medical Center, Columbus, OH; ${ }^{5}$ Department of \\ Internal Medicine, Division of Medical Oncology, The Ohio State University Wexner Medical Center, Columbus, OH; \\ ${ }^{6}$ Department of Otolaryngology-Head \& Neck Surgery, Washington University School of Medicine, Ear, Nose \& Throat \\ Center, St. Louis, MO
}

\begin{abstract}
Background. The COVID-19 pandemic has required triage and delays in surgical care throughout the world. The impact of these surgical delays on survival for patients with head and neck squamous cell carcinoma (HNSCC) remains unknown.

Methods. A retrospective cohort study of 37730 patients in the National Cancer Database with HNSCC who underwent primary surgical management from 2004 to 2016 was performed. Uni- and multivariate analyses were used to identify predictors of overall survival. Bootstrapping methods were used to identify optimal time-to-surgery (TTS) thresholds at which overall survival differences were greatest. Cox proportional hazard models with or without restricted cubic splines were used to determine the association between TTS and survival.
\end{abstract}

C.J. Rygalski and S. Zhao: These authors contributed equally to this manuscript

Electronic supplementary material The online version of this article (https://doi.org/10.1245/s10434-020-09326-4) contains supplementary material, which is available to authorized users.

(C) Society of Surgical Oncology 2020

First Received: 19 June 2020

Accepted: 16 September 2020;

Published Online: 13 November 2020

S. Y. Kang, MD

e-mail: Stephen.kang@osumc.edu
Results. The study identified TTS as an independent predictor of overall survival (OS). Bootstrapping the data to dichotomize the cohort identified the largest rise in hazard ratio (HR) at day 67 , which was used as the optimal TTS cut-point in survival analysis. The patients who underwent surgical treatment longer than 67 days after diagnosis had a significantly increased risk of death (HR, 1.189; 95\% confidence interval [CI], 1.122-1.261; $P<0.0001)$. For every 30-day delay in TTS, the hazard of death increased by $4.6 \%$. Subsite analysis showed that the oropharynx subsite was most affected by surgical delays, followed by the oral cavity.

Conclusions. Increasing TTS is an independent predictor of survival for patients with HNSCC and should be performed within 67 days after diagnosis to achieve optimal survival outcomes.

The novel 2019 coronavirus disease (COVID-19) pandemic has led to delays in the surgical care of patients with head and neck squamous cell carcinoma (HNSCC) Accordingly, the current climate has raised urgent questions for patients as well as the head and neck oncologic community. How long can surgery be delayed, and at what time point does further delay have a significant impact on survival outcomes?

Previous study has shown that the time from diagnosis to initiation of definitive treatment for HNSCC is increasing, adversely affecting survival. ${ }^{1-5}$ Treatment package times, which cover the time from diagnosis to completion 
of treatment including adjuvant therapy, also have correlated with survival. ${ }^{5-10}$ Although these data collectively show the deleterious effect of delayed treatment on patients, both surgically and nonsurgically, current events have demonstrated a need to study the specific impact of surgical delay on HNSCC survival so that surgeons can appropriately schedule surgeries while balancing risks and vital resources.

From a large national database of patients with HNSCC, this study included only patients treated with primary surgery. The study aimed to analyze the impact of increasing time to surgery (TTS) on overall survival (OS) in HNSCC.

\section{MATERIALS AND METHODS}

\section{Data Source}

The National Cancer Database (NCDB) is a hospitalbased cancer registry database that collects data from more than 1500 Commission on Cancer-accredited facilities. The NCDB database captures more than $80 \%$ of newly diagnosed head and neck cancers each year in the United States. ${ }^{11}$ We reviewed the NCDB for patients from 2004 to 2016 with primary oral cavity, oropharynx, larynx, or hypopharynx squamous cell carcinoma (SCC) who received definitive primary surgical treatment and investigated the effect of TTS on OS. The Ohio State University College of Medicine Institutional Review Board deemed this study exempt from review.

\section{Inclusion and Exclusion Criteria}

A retrospective review of the NCDB was performed for adults with previously untreated SCC of the oral cavity, oropharynx, larynx, or hypopharynx diagnosed between 2004 and 2016 (Fig. S1). Squamous cell carcinoma was identified with International Classification of Diseases for Oncology, third edition, histologic codes 8052, 8070-8076, 8078,8083 , and 8084 . Only entries with subsite information were included, and the subsites were grouped by International Classification of Diseases for Oncology-3 codes.

The oral cavity primary site codes included $\mathrm{C} 02.2$, C02.3, C03.0, C03.1, C03.9, C04.0, C04.1, C04.8, C04.9, C05.0, C06.0, C06.1, and C06.2. ${ }^{12}$ The oropharynx primary site codes included C01.9, C02.4, C05.1, C05.2, C09.0, $\mathrm{C} 09.1, \mathrm{C} 09.8, \mathrm{C} 09.9, \mathrm{C} 10.2, \mathrm{C} 10.3$, and $\mathrm{C} 10.9 .{ }^{13}$ The hypopharynx primary site codes included C12.9, C13.0, $\mathrm{C} 13.1, \mathrm{C} 13.2, \mathrm{C} 13.8$, and $\mathrm{C} 13.9$. The larynx primary site codes included C10.1, C32.1, C32.0, C32.2, C32.8 and C32.9. In the subset analysis of the oropharynx, patients with documented high-risk human papilloma virus (HPV), including HPV-16, HPV-18, and high-risk HPV not otherwise specified [NOS]), were considered positive for HPV.

Tumors involving the lip were excluded due to the risk of misclassification from skin cancers. Subsite codes including tongue NOS (C02.9), overlapping tongue lesion (C02.8), border of tongue (C02.1), and overlapping lesion of the mouth (C06.8) were excluded because these may represent either oral cavity or oropharyngeal subsites, and subsite analysis was an important covariate in this study.

\section{Defining Definitive Primary Surgery in the NCDB}

We sought to further filter the aforementioned cohort to ensure that patients included in this study underwent definitive primary surgery. ${ }^{13,14}$ Patients who had undergone preoperative chemotherapy, radiation therapy, or both were excluded. To ensure that patients received definitive surgery, the primary-site surgical codes from the Surveillance, Epidemiology, and End Results (SEER) Program Code Manual (3rd edition, revision 1) then were selected for each subsite that were unlikely to denote diagnostic biopsies. ${ }^{15}$ The codes for oral cavity are $30,40,41,42$, and 43 , and the codes for the oropharynx are $30,31,32,40,41$, $42,43,50,51$, and 52 . Due to concerns that a proportion of patients classified as 30 or 31 did not receive definitive surgery, patients were included only if they also had more than 10 lymph nodes examined during surgery. ${ }^{13}$ The larynx codes were $30,31,32,33,40,41,42,50$ and 80 . The hypopharynx codes were $32,40,41,42,43,50,51$, and 52 . The patients who had multiple primary cancers or distant metastatic disease treated with palliative intent were excluded (Fig. S1).

\section{Variables}

The covariates for the survival analyses were age, TTS, sex, race, insurance, urban residence, facility type, Charlson-Deyo score, income, education level, surgical margin status, pathologic stage, adjuvant treatment, and primary site. The TTS is defined as the number of days between the date of diagnosis, which is the first date the cancer is either clinically described in the medical record or histologically confirmed, and the date of the most definitive surgical procedure performed on the primary site. The patients with an interval of 0 days from diagnosis to definitive surgery were excluded due to accuracy concerns. ${ }^{2}$ Intervals longer than 180 days also were excluded from the analysis due to concerns of bias if these patients did not receive up-to-date imaging to rule out distant metastases. 


\section{Statistical Methods}

Descriptive statistics were used to characterize the overall study population using median and interquartile range for continuous variables or frequencies and proportions for categorical variables. The primary outcome of this study was OS, determined from the date of definitive surgery to death or last follow-up visit.

Univariate analysis was used to identify potential predictors for death. Variables with a $p$ value lower than 0.10 were entered into a multivariable model. Variables were removed sequentially from the multivariable model using the backward selection method, based upon $p$ value. Cox proportional hazard models with or without restricted cubic splines were used to determine the association between TTS and survival, with control for the significant predictors from backward selection. An optimal threshold for TTS was determined by taking 100 bootstrap samples of the data and calculating adjusted hazard ratios (HRs) for each cut-point of TTS between 50 and 90 days. Locally estimated scatterplot smoothing was used to visualize the overall trend in TTS thresholds compared with adjusted HRs. Adjusted survival curves were plotted by optimal TTS thresholds. Interactions of TTS with stage, surgical margins, and tumor subsite were tested, and significant interactions were visualized using lattice plots. All $p$ values lower than 0.05 were considered statistically significant. To plot restricted cubic splines, $\mathrm{R}$ statistical software (version 3.6.0) (R Foundation for Statistical Computing, Vienna, Austria) was used, and SAS (9.4) (Cary, NC) was used for the remainder of the analysis.

\section{RESULTS}

\section{Baseline Population Demographics}

Baseline demographics are shown in Table 1. The median TTS in the United States for HNSCC from 2004 to 2016 was 33 days (IQR, 21- 49 days). The TTS was 30 days for $45 \%$ of the patients, 30 to 60 days for $41 \%$ of the patients, and longer than 60 days for $14 \%$ of the patients. The most frequent subsite was the oral cavity (49\%), followed by the oropharynx (28\%), larynx (20\%), and hypopharynx $(2 \%)$.

\section{Increasing TTS and Survival}

An adjusted cubic spline was used to assess the relationship between TTS and survival given that we did not want to assume it was a simple linear association (Fig. 1a). This showed that the HR for death was lowest near day 24, with a gradual rise in the HR of death after day 40 and beyond, even when control was used for pathologic stage,
TABLE 1 Baseline characteristics of patients with head and neck squamous cell carcinoma treated with primary surgery $(n=37730)$

\begin{tabular}{|c|c|}
\hline Characteristic & $N(\%)$ \\
\hline \multicolumn{2}{|l|}{ Age at diagnosis } \\
\hline Median (IQR) & $60(53-68)$ \\
\hline \multicolumn{2}{|l|}{ TTS (days) } \\
\hline Median (IQR) & $33(21-49)$ \\
\hline $1-30$ & $16957(45)$ \\
\hline $30-60$ & $15348(41)$ \\
\hline $60-90$ & $3881(10)$ \\
\hline $91+$ & $1544(4)$ \\
\hline \multicolumn{2}{|l|}{ Great circle distance } \\
\hline Median (IQR) & $18.1(7.1-47.9)$ \\
\hline \multicolumn{2}{|l|}{ Sex } \\
\hline Male & $26833(71)$ \\
\hline Female & $10897(29)$ \\
\hline \multicolumn{2}{|l|}{ Race $(n=37311)$} \\
\hline White & $32978(88)$ \\
\hline Black & $3140(8)$ \\
\hline Other & $1193(3)$ \\
\hline \multicolumn{2}{|l|}{ Median household income $(n=37082)$} \\
\hline$<\$ 40227$ & $7539(20)$ \\
\hline$\$ 40227-50353$ & $9010(24)$ \\
\hline$\$ 50354-63332$ & $8616(23)$ \\
\hline$\geq \$ 63333$ & $11917(32)$ \\
\hline \multicolumn{2}{|l|}{ Percent with no high school degree $(n=37$ 186) } \\
\hline$\geq 17.6$ & $7943(21)$ \\
\hline $10.9-17.5$ & $10210(27)$ \\
\hline $6.3-10.8$ & $10456(28)$ \\
\hline$<6.3$ & $8577(23)$ \\
\hline \multicolumn{2}{|l|}{ Insurance status $(n=36570)$} \\
\hline Not insured & $2042(6)$ \\
\hline Private insurance & $16464(45)$ \\
\hline Public insurance & 18064 (49) \\
\hline \multicolumn{2}{|l|}{ Urban status $(n=36853)$} \\
\hline Nonmetropolitan & $7218(20)$ \\
\hline Metropolitan & $29635(80)$ \\
\hline \multicolumn{2}{|l|}{ Facility type $(n=36651)$} \\
\hline Community cancer program & $1491(4)$ \\
\hline Comprehensive community cancer program & $8668(24)$ \\
\hline Academic/research program & $23110(63)$ \\
\hline Integrated network cancer program & $3382(9)$ \\
\hline \multicolumn{2}{|l|}{ Charlson-Deyo score } \\
\hline 0 & $28028(74)$ \\
\hline 1 & 7297 (19) \\
\hline 2 & $1746(5)$ \\
\hline $3+$ & $659(2)$ \\
\hline \multicolumn{2}{|l|}{ Surgical margin status $(n=35902)$} \\
\hline Negative & $30244(84)$ \\
\hline Positive & $5658(16)$ \\
\hline
\end{tabular}


TABLE 1 continued

\begin{tabular}{ll}
\hline Characteristic & $N(\%)$ \\
\hline TNM pathologic stage $(n=32$ 394) & \\
1 & $5112(16)$ \\
2 & $3677(11)$ \\
3 & $5183(16)$ \\
4 & $18422(57)$ \\
Adjuvant treatment & \\
No adjuvant treatment & $16451(44)$ \\
Adjuvant radiation & $9860(26)$ \\
Adjuvant chemoradiation & $11419(30)$ \\
Primary site & \\
Oral cavity & $18672(49)$ \\
Oropharynx & $10697(28)$ \\
Larynx & $7641(20)$ \\
Hypopharynx & $720(2)$
\end{tabular}

$I Q R$ interquartile range, TTS time to surgery; TNM tumor-nodemetastasis

surgical margins, primary site, adjuvant treatment, age, sex, race, income, insurance status, and Charlson-Deyo score. Bootstrapping the data determined an optimal threshold for dichotomizing TTS based on an OS of 67 days (Fig. 2a) . Adjusted OS is demonstrated in Fig. 2b, showing that a TTS longer than 67 days independently predicted a shorter OS than surgery performed within 67 days after diagnosis (HR, 1.189; 95\% CI, 1.122-1.261).
The uni- and multivariate analyses are shown in Table 2. In the multivariable analysis, longer TTS was significantly associated with worse OS even after control for age, sex, race, income level, insurance, distance to the hospital, Charlson-Deyo score, pathologic stage, surgical margins, adjuvant treatment, and primary site. For each increase in TTS by 30 days, the hazard of death increased by $4.6 \%$ (eTable S1).

\section{Interaction of TTS With Stage, Charlson-Deyo Score, Surgical Margins, and Tumor Subsite}

Interaction testing was performed to assess mortality risk according to TTS by overall stage group, CharlsonDeyo score, surgical margins, and tumor subsite. No significant interactions were found between TTS and American Joint Committee on Cancer (AJCC) stage $(p=0.3870)$ or between TTS and Charlson-Deyo score $(p=0.2694)$. However, a significant interaction between TTS and tumor subsite was identified $(p \leq 0.0001)$ as well as surgical margin status $(p=0.0076)$. Adjusted restricted cubic spline analysis by tumor subsite was performed, and the results are presented in Fig. 1a-d.

The association of TTS with mortality was observed at the earliest time point in the oropharynx subsite, followed closely by the oral cavity. A relatively later time point of association between TTS and survival was observed in the larynx/hypopharynx subsite (Fig. 1d). Within the oropharynx subset, we also performed an analysis,
FIG. 1 Relationship of time to surgery and hazard ratio (HR) of death. a Adjusted restricted cubic spline shows that the HR for the overall cohort was lowest on day 24 , with the HR steadily increasing with time after day 40. Cubic splines for the oral cavity, oropharynx, and larynx/hypopharynx subsites are shown in $\mathbf{b}, \mathbf{c}$, and $\mathbf{d}$, respectively
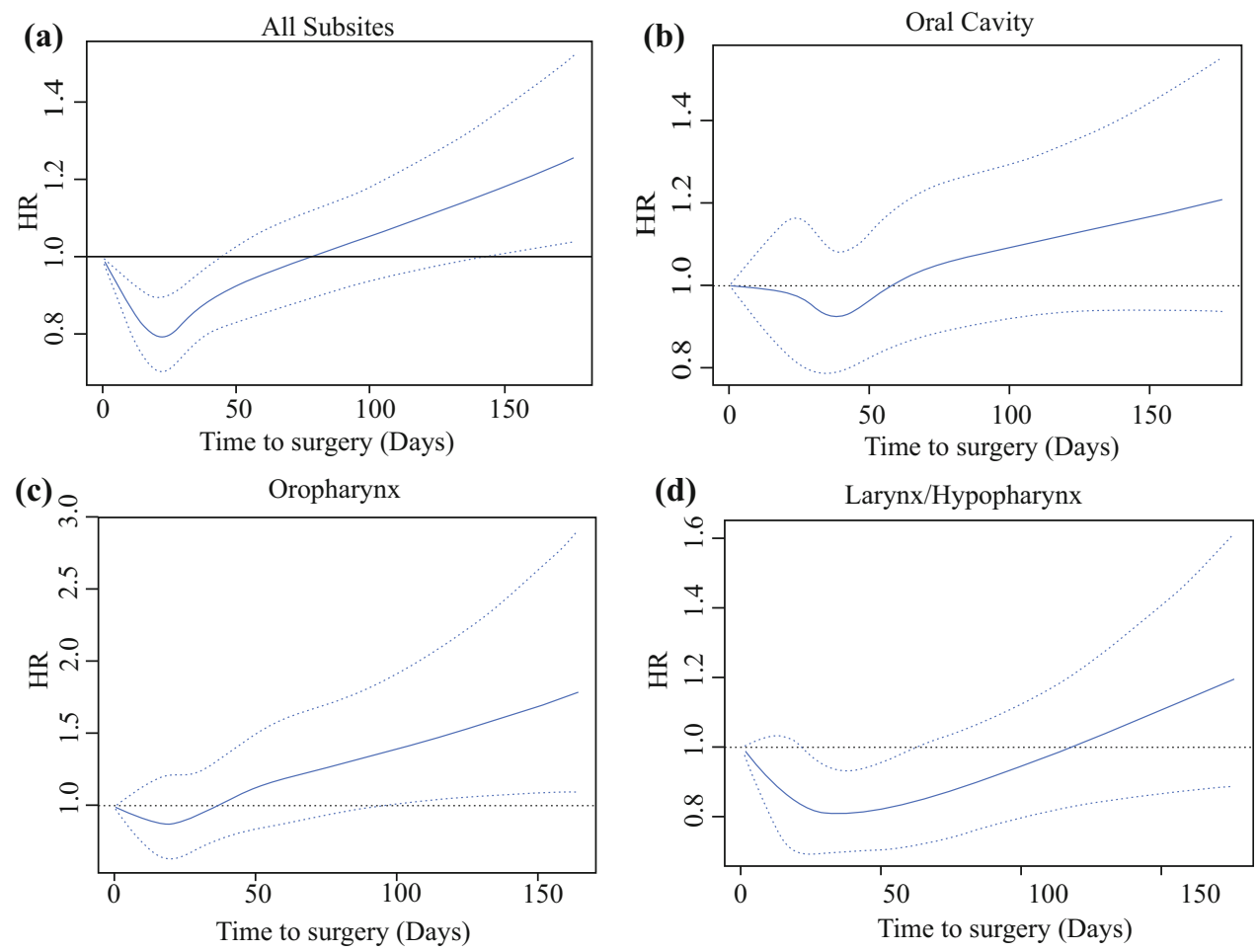
(a)

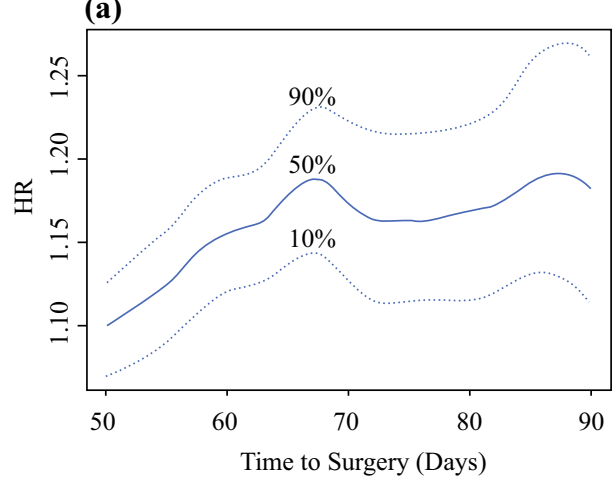

FIG. 2 Cohort validation and survival analysis. a The threshold for time to surgery (TTS) was determined by 100 bootstrap samples of the data and calculation of adjusted hazard ratios (HRs) for each cutpoint of TTS between 50 and 90 days. Locally estimated scatterplot smoothing shows the overall trend in TTS thresholds versus adjusted

stratifying by HPV-positive and HPV-negative tumors, which showed no interaction between TTS and survival $(p=0.93)$.

The interaction between tumor subsite and TTS was further explored by categorizing TTS into 0 to 30 days, 31 to 60 days, 61 to 90 days, and longer than 90 days. Hazard ratios stratified by tumor subsite and TTS for unadjusted tumor stage and surgical margin status, partial adjustment for these factors (model M1), and full adjustment for all significant covariates (model M2) are shown in Fig. 3. Unadjusted HRs for TTS categories relative to the baseline category (0-30 days) were significant for both oral cavity and oropharynx subsites, but not the larynx/hypopharynx. After adjustment for tumor stage and surgical margin status, HRs for TTS in oral cavity cancers were reduced by approximately half but remained significant for 61 to 90 days (HR, 1.18 ; 95\% CI, 1.09-1.28) and longer than 90 days (HR, $1.19 ; 95 \%$ CI, 1.07-1.34) compared with 0 to 30 days. In the oral cavity, HRs were further reduced for TTS after adjustment for all additional significant covariates (61-90 vs $0-30$ days: HR, $1.09 ; 95 \%$ CI, $1.00-1.18 ;>90$ vs $0-30$ days: HR, $1.10 ; 95 \%$ CI, 0.98-1.23). Conversely, the HRs for TTS in the oropharynx were relatively unchanged even in the fully adjusted model (31-60 vs $0-30$ days: HR, 1.29; 95\% CI, $1.14-1.46$; 61-90 vs $0-30$ days: HR, $1.47 ; 95 \%$ CI, $1.21-1.79 ;>90$ days vs 0-30 days: HR, 1.98 ; 95\% CI, 1.44-2.73).

\section{DISCUSSION}

In the United States, TTI in HNSCC, including surgical and nonsurgical methods, is on the rise and associated with concurrent chemoradiation treatment, treatment at academic facilities, advanced-stage disease, and transitions in treatment. ${ }^{1}$ Murphy et al. $^{2}$ found that an increase in TTI

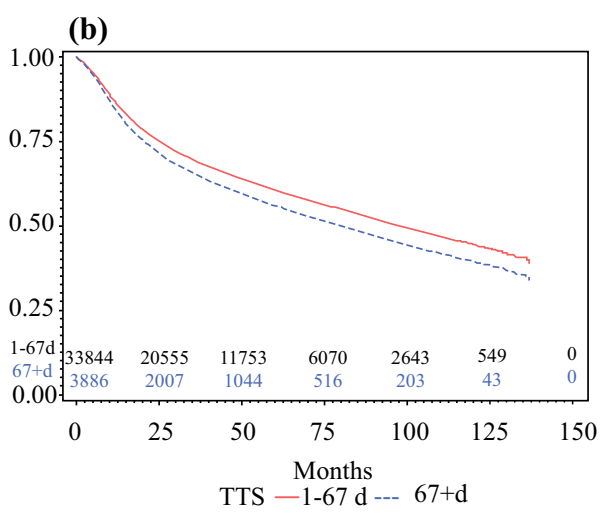

HRs indicating a peak at 67 days. b Adjusted overall survival showing that patients undergoing surgery longer than 67 days after diagnosis have a poorer overall survival than those undergoing surgery within 67 days after diagnosis (HR, 1.189; 95\% confidence interval [CI], 1.122-1.261; $p<0.0001)$

was an independent predictor of survival, and that a threshold of TTI longer than 46 to 52 days introduced the increased risk of death, which was most detrimental at a TTI longer than 67 days.

Important differences exist between the current study and the paper published by Murphy et al. ${ }^{2}$ The cohort described by Murphy et $\mathrm{al}^{2}$ was composed of patients receiving both nonsurgical (53\%) and primary surgical $(44 \%)$ treatment with or without adjuvant radiation or chemoradiation therapy, and $2.5 \%$ of their cohort received adjuvant chemotherapy, preoperative radiation therapy, preoperative CRT, or preoperative chemotherapy. ${ }^{2}$

Additional differences in the study populations exist, with the study by Murphy et al. ${ }^{2}$ composed mostly of laryngeal cancers (42\%), followed by oral cavity (29\%), oropharynx (22\%), and hypopharynx (7\%) cancers, likely as a result of including surgical and nonsurgical treatment methods. ${ }^{2}$ The current study was composed mostly of oral cavity cancers (49\%), followed by oropharynx (28\%), larynx (20\%), and hypopharynx (2\%) cancers. Murphy et al. ${ }^{2}$ performed a subset analysis of the patients in their cohort treated with surgery alone, which showed an increased risk of death in delays longer than 60 days. $^{2}$ Despite differences in study populations, our findings also show a significant association with surgical delay and increased mortality, with the largest increase in HR at day 67.

Ho et al. ${ }^{5}$ investigated the impact of radiation duration, radiation delays, and surgical delays on OS for patients undergoing primary surgery and adjuvant radiation or chemoradiation treatment. The findings showed that prolonged radiation intervals and time to radiation were associated with overall mortality, whereas surgical delays did not increase the risk of mortality. ${ }^{5}$ Because the goal was collective investigation of surgical delays, radiation 
TABLE 2 Uni- and multivariate analysis of factors associated with overall survival

\begin{tabular}{|c|c|c|c|c|c|c|}
\hline \multirow[b]{3}{*}{ Characteristic } & \multicolumn{3}{|c|}{ Univariate analysis } & \multicolumn{3}{|c|}{ Multivariate analysis } \\
\hline & \multirow[b]{2}{*}{$\mathrm{HR}$} & \multicolumn{2}{|l|}{$95 \%$ CI } & \multirow[b]{2}{*}{ HR } & \multicolumn{2}{|c|}{$95 \%$ CI } \\
\hline & & Lower & Upper & & Lower & Upper \\
\hline Age & 1.033 & 1.032 & 1.035 & 1.022 & 1.02 & 1.024 \\
\hline Great circle distance per 10 miles & 0.999 & 0.997 & 1.00 & 0.998 & 0.996 & 1.00 \\
\hline \multicolumn{7}{|l|}{ Sex } \\
\hline Male & Ref & - & - & Ref & - & - \\
\hline Female & 1.037 & 1.00 & 1.077 & 0.873 & 0.836 & 0.912 \\
\hline \multicolumn{7}{|l|}{ Race } \\
\hline White & Ref & - & - & Ref & - & - \\
\hline Black & 1.44 & 1.363 & 1.521 & 1.06 & 0.994 & 1.131 \\
\hline Other & 0.965 & 0.869 & 1.07 & 0.9 & 0.801 & 1.011 \\
\hline \multicolumn{7}{|l|}{ Median household income } \\
\hline$<\$ 40227$ & 1.59 & 1.516 & 1.669 & 1.196 & 1.130 & 1.266 \\
\hline$\$ 40227-50353$ & 1.412 & 1.347 & 1.48 & 1.157 & 1.097 & 1.220 \\
\hline$\$ 50354-63332$ & 1.264 & 1.204 & 1.327 & 1.128 & 1.068 & 1.191 \\
\hline$\geq \$ 63333$ & Ref & - & - & Ref & - & - \\
\hline \multicolumn{7}{|l|}{ Percent with no high school degree } \\
\hline$\geq 17.6$ & 1.557 & 1.478 & 1.641 & - & - & - \\
\hline $10.9-17.5$ & 1.367 & 1.3 & 1.438 & - & - & - \\
\hline $6.3-10.8$ & 1.235 & 1.174 & 1.3 & - & - & - \\
\hline$<6.3$ & Ref & - & - & - & - & - \\
\hline \multicolumn{7}{|l|}{ Insurance } \\
\hline Not insured & 0.781 & 0.726 & 0.84 & 0.981 & 0.903 & 1.065 \\
\hline Private insurance & 0.429 & 0.413 & 0.445 & 0.708 & 0.675 & 0.743 \\
\hline Public insurance & Ref & & & Ref & & \\
\hline \multicolumn{7}{|l|}{ Urban status } \\
\hline Nonmetropolitan & Ref & - & - & - & - & - \\
\hline Metropolitan & 0.897 & 0.86 & 0.935 & - & - & - \\
\hline \multicolumn{7}{|l|}{ Facility type } \\
\hline Community & Ref & - & - & - & - & - \\
\hline Comprehensive Community & 0.905 & 0.83 & 0.988 & - & - & - \\
\hline Academic/research & 0.925 & 0.851 & 1.006 & - & - & - \\
\hline I ntegrated network & 0.922 & 0.836 & 1.018 & - & - & - \\
\hline \multicolumn{7}{|l|}{ Charlson-Deyo score } \\
\hline 0 & Ref & - & - & Ref & - & - \\
\hline 1 & 1.498 & 1.439 & 1.56 & 1.2135 & 1.161 & 1.272 \\
\hline 2 & 1.953 & 1.824 & 2.092 & 1.485 & 1.375 & 1.603 \\
\hline $3+$ & 2.311 & 2.084 & 2.563 & 1.674 & 1.492 & 1.878 \\
\hline \multicolumn{7}{|l|}{ Surgical margins } \\
\hline Negative & Ref & - & - & Ref & - & - \\
\hline Positive & 1.39 & 1.33 & 1.452 & 1.414 & 1.346 & 1.486 \\
\hline \multicolumn{7}{|l|}{ TNM pathologic stage } \\
\hline 1 & Ref & - & - & Ref & - & - \\
\hline 2 & 1.432 & 1.317 & 1.557 & 1.469 & 1.345 & 1.604 \\
\hline 3 & 1.614 & 1.496 & 1.741 & 2.148 & 1.977 & 2.333 \\
\hline 4 & 2.388 & 2.243 & 2.543 & 3.15 & 2.929 & 3.388 \\
\hline
\end{tabular}


TABLE 2 continued

\begin{tabular}{|c|c|c|c|c|c|c|}
\hline \multirow[b]{3}{*}{ Characteristic } & \multicolumn{3}{|c|}{ Univariate analysis } & \multicolumn{3}{|c|}{ Multivariate analysis } \\
\hline & \multirow[b]{2}{*}{ HR } & \multicolumn{2}{|l|}{$95 \% \mathrm{CI}$} & \multirow[b]{2}{*}{ HR } & \multicolumn{2}{|l|}{$95 \% \mathrm{CI}$} \\
\hline & & Lower & Upper & & Lower & Upper \\
\hline \multicolumn{7}{|l|}{ Adjuvant treatment } \\
\hline no adjuvant treatment & Ref & - & - & Ref & - & - \\
\hline Adjuvant radiation & 1.058 & 1.014 & 1.103 & 0.776 & 0.738 & 0.816 \\
\hline Adjuvant chemoradiation & 1.037 & 0.996 & 1.079 & 0.9 & 0.854 & 0.949 \\
\hline \multicolumn{7}{|l|}{ Primary sites } \\
\hline Oral cavity & Ref & - & - & Ref & - & - \\
\hline Oropharynx & 0.406 & 0.386 & 0.427 & 0.368 & 0.347 & 0.39 \\
\hline Larynx & 1.128 & 1.084 & 1.174 & 0.854 & 0.814 & 0.896 \\
\hline Hypopharynx & 1.834 & 1.667 & 2.018 & 1.201 & 1.08 & 1.336 \\
\hline \multicolumn{7}{|l|}{ TTS (days) } \\
\hline $1-67$ & Ref & Ref & - & Ref & - & - \\
\hline $67+$ & 1.371 & 1.302 & 1.444 & 1.189 & 1.122 & 1.261 \\
\hline
\end{tabular}

$H R$ hazard ratio, $C I$ confidence interval, TNM tumor-node-metastasis, TTS time to surgery

delays, and radiation duration and their impact on survival, the study included only patients receiving surgery and adjuvant therapy. ${ }^{5}$ Patients who received surgery alone were excluded, which may have resulted in selection of a

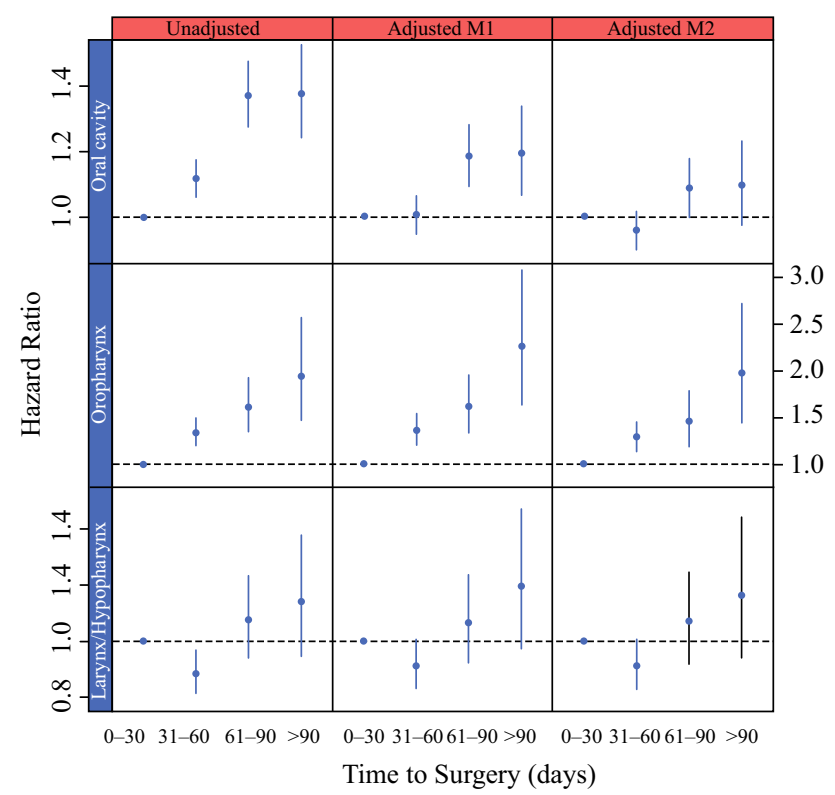

FIG. 3 Relationship of time to surgery (TTS) and hazard ratio of death by tumor subsite. Hazard ratio is shown by tumor subsite in the unadjusted, adjusted M1 (partially adjusted for surgical margins and tumor stage only), and adjusted M2 (fully adjusted) models. The oropharynx subsite showed greater mortality when surgery was performed beyond 30 days, whereas the oral cavity subsite showed greater mortality when surgery was performed beyond 60 days. Control for surgical margins and tumor stage in the oral cavity subsite caused a $50 \%$ reduction in hazard ratios for TTS in the partially adjusted model different cohort than the current study, which included all patients undergoing primary surgical treatment with or without adjuvant treatment. Our cohort was comprised of 16451 patients (44\%) treated with surgery alone. This study population was selected to investigate the impact of TTS on OS for external validity purposes because it is often difficult to discern preoperatively whether adjuvant therapy will be indicated.

Xiao et $\mathrm{al}^{4}$ showed that surgical delays are associated with clinical-to-pathologic upstaging. These authors also showed that compared with a TTS of 1 to 6 days, increasing TTS significantly predicts T upstaging, even for intervals as short as 7 to 13 days. ${ }^{4}$ They also showed that patients experiencing tumor progression and clinical-topathologic upstaging had significantly poorer survival than the patients who did not experience tumor progression, solidifying tumor progression as a mechanism of mortality in the surgically delayed HNSCC population. ${ }^{4}$

After adjustment for all known confounders, our analysis showed that surgical delays longer than 67 days independently predicted increased risk of death (HR, 1.189; 95\% CI, 1.122-1.261). Delays beyond this time point resulted in poorer survival that cannot be accounted for solely by factors such as tumor stage, positive margin rates, medical comorbidities, and insurance status. Because the current study adjusted for patients by pathologic staging as determined at the time of surgery, the survival difference also is not explained by pathologic upstaging. When the analysis was performed with the TTS variable grouped in 30-day increments, with each increase in TTS by 30 days, the hazard of death increased by $4.6 \%$ (eTable S1). 
Primary surgical treatment longer than 67 days after diagnosis increases the risk of mortality by $18.9 \%$. Although the magnitude of the HR is relatively small, the strength of the findings exist in the ability of the treating team to modify the TTS covariate, compared with other tumor-intrinsic factors such as tumor stage, subsite, and HPV status.

When analyzed by subsite, the oropharynx showed the greatest association with increasing TTS, and this association did not vary by HPV status. These data suggest that oropharyngeal surgery wait times should be minimized when possible because wait times longer than 30 days showed a $29 \%$ increase in mortality relative to surgery performed within 30 days. Oral cavity cancers also showed a significant increase in mortality with surgical delays, although this was noted at a later time point, when surgery was delayed longer than 60 days. In contrast, the larynx/ hypopharynx subsites showed the least association between OS and TTS (Fig. 1D), potentially due to protection from local tumor progression offered from the cartilaginous framework of the larynx.

To investigate the potential impact of increasing pathologic stage and rate of positive surgical margins with increasing TTS, we also created a partially adjusted model (Fig. 3, model M1) that adjusted only for stage and surgical margins. This showed a $50 \%$ reduction in $\mathrm{HR}$ in the oral cavity subsite compared with the unadjusted model at 61 days, suggesting that increasing pathologic stage and surgical margins are additional negative prognosticators associated with surgical delay in the oral cavity subsite that are not conveyed by the HR in the fully adjusted model. Conversely, the oropharynx subsite showed little reduction in the HR when control was used for stage and surgical margins, and thus delays in this cohort resulted in increased mortality that could not be explained by stage progression or increased positive margins.

One potential mechanism whereby increased TTS affected survival in our fully adjusted model was the potential for increased risk of distant metastasis for patients with longer TTS undetected at the time of diagnosis and surgery that became clinically apparent later in the treatment course. Additionally, with longer surgical delays, patients are potentially at greater risk of perineural invasion, lymphovascular invasion, and greater depth of invasion, all of which are not measured in this NCDB cohort.

Previous studies have shown an association between delayed postoperative radiation and increased mortality. ${ }^{6,7}$ Additionally, total treatment package time is associated with mortality, particularly with package lengths longer than 100 days. ${ }^{5,8,16}$ Treatment package time should be distinguished from TTS because treatment package time includes key variables out of surgeons' control. These include dental and preoperative clearance, coordination/ availability of medical oncology and radiation oncology appointments, delayed pathology results, and prolonged wound healing. Under normal circumstances, relatively fewer barriers are present with regard to TTS (e.g., timely surgical evaluation, performance of biopsies, operating room time, and availability), which remain more closely associated with surgeons' purview.

In clinical practice, TTS often is balanced by the perioperative optimization process (i.e., adequate nutrition, tobacco and alcohol cessation, medical clearance, imaging), which is critical for patient treatment. Patients with greater comorbidities are likely at risk for greater surgical delay, and our analysis showed significant differences in TTS among Charlson-Deyo score categories $(p<0.0001)$. As a result, we controlled for Charlson-Deyo scores in our multivariable model and also tested the interaction between TTS and Charlson-Deyo scores in the survival model, which was not significant ( $p=0.2694)$, suggesting that the association between TTS and OS did not differ significantly across Charlson-Deyo score categories.

These results raise an important question: Should TTS be implemented as an institutional quality metric? Although it has been shown that high-volume centers offer specialized care with better outcomes than low-volume institutions, they also are potentially associated with longer wait times. $^{2,17-21}$ Through institutional TTS tracking initiatives, it may be possible to describe more clearly the individual components that result in prolonged TTS and serve better to inform future quality improvement projects. Although TTS has been considered as a quality measure at other sites, it has yet to be considered in HNSCC, perhaps partly due to a lack of clear data to support such a measure. $^{22}$ Although establishing TTS as a quality metric at this time is not a priority, these and other data suggest that TTS should be considered as a quality metric in the future.

The current study had important limitations. Due to the multidisciplinary nature of HNSCC management, TTS is relevant only for patients managed with primary surgery. Although challenging to achieve in the NCDB, we placed increased importance on isolating a primary surgical cohort as outlined in the Methods section. Despite every effort to adjust for numerous variables included in the database, unmeasured confounders still could exist as variables such as tobacco and alcohol exposure that are not recorded in the NCDB. More data are needed because the NCDB does not record disease-free or disease-specific survival. Because the NCDB records the date of diagnosis as either the first day that the cancer is mentioned in the record or the date of histologic diagnosis, variability between patients exists. Additionally, the authors recognize that significant heterogeneity exists among mucosal HNSCCs, and although understanding tumor growth could assist in 
triaging of surgical cases, these factors are difficult to discern at the time of initial evaluation. Biomarkers or other predictors of tumor growth rates could potentially further define the urgency for timely surgery and individualized wait times. A prospective trial designed to randomize patients receiving surgical treatment to differing wait times and TTS is neither ethical nor feasible. Therefore, we believe this analysis of the NCDB provides the most timely and robust data available.

\section{CONCLUSIONS}

Increasing TTS independently predicts worse survival for patients with HNSCC. The patients in this study who had TTS longer than 67 days were independently predicted to experience worse overall survival than the patients with TTS of 67 days or less. Although surgical delays are inevitable during the COVID-19 pandemic, all reasonable efforts should be made to expedite primary surgery for HNSCC, especially in the oropharynx and oral cavity subsites.

DISCLOSURE Antoine Eskander MD, ScM, is a paid consultant for Bristol-Myers and has received research support from Merck. The remaining authors have no conflicts of interest.

\section{REFERENCES}

1. Murphy CT, Galloway TJ, Handorf EA, et al. Increasing time to treatment initiation for head and neck cancer: an analysis of the National Cancer Database. Cancer. 2015;121:1204-13. https://d oi.org/10.1002/cncr.29191.

2. Murphy CT, Galloway TJ, Handorf EA, et al. Survival impact of increasing time to treatment initiation for patients with head and neck cancer in the United States. J Clin Oncol. 2016;34:169-78. https://doi.org/10.1200/jco.2015.61.5906.

3. Cheraghlou S, Kuo P, Judson BL. Treatment delay and facility case volume are associated with survival in early-stage glottic cancer. Laryngoscope. 2017;127:616-22. https://doi.org/10.1002/ lary.26259.

4. Xiao R, Ward MC, Yang K, et al. Increased pathologic upstaging with rising time to treatment initiation for head and neck cancer: a mechanism for increased mortality. Cancer. 2018;124:1400-14. https://doi.org/10.1002/cncr.31213.

5. Ho AS, Kim S, Tighiouart M, et al. Quantitative survival impact of composite treatment delays in head and neck cancer. Cancer. 2018;124:3154-62. https://doi.org/10.1002/cncr.31533.

6. Harris JP, Chen MM, Orosco RK, Sirjani D, Divi V, Hara W. Association of survival with shorter time to radiation therapy after surgery for U.S. patients with head and neck cancer. JAMA Otolaryngol Head Neck Surg. 2018;144:349-59. https://doi.org/ 10.1001/jamaoto.2017.3406

7. Graboyes EM, Garrett-Mayer E, Ellis MA, et al. Effect of time to initiation of postoperative radiation therapy on survival in surgically managed head and neck cancer. Cancer. 2017;123:4841-50. https://doi.org/10.1002/cncr.30939.

8. Goel AN, Frangos MI, Raghavan G, et al. The impact of treatment package time on survival in surgically managed head and neck cancer in the United States. Oral Oncol. 2019;88:39-48. h ttps://doi.org/10.1016/j.oraloncology.2018.11.021.

9. Graboyes EM, Kompelli AR, Neskey DM, et al. Association of treatment delays with survival for patients with head and neck cancer: a systematic review. JAMA Otolaryngol Head Neck Surg. 2019;145:166-77. https://doi.org/10.1001/jamaoto.2018.2716.

10. Fujiwara RJT, Judson BL, Yarbrough WG, Husain Z, Mehra S. Treatment delays in oral cavity squamous cell carcinoma and association with survival. Head Neck. 2017;39:639-46. https://d oi.org/10.1002/hed.24608.

11. Bilimoria KY, Stewart AK, Winchester DP, Ko CY. The National Cancer Data Base: a powerful initiative to improve cancer care in the United States. Ann Surg Oncol. 2008;15:683-90. https://doi. org/10.1245/s10434-007-9747-3.

12. Zhan KY, Morgan PF, Neskey DM, et al. Preoperative predictors of occult nodal disease in cT1N0 oral cavity squamous cell carcinoma: review of 2623 cases. Head Neck. 2018;40:1967-76. h ttps://doi.org/10.1002/hed.25178.

13. Zhan KY, Puram SV, Li MM, et al. National treatment trends in human papillomavirus-positive oropharyngeal squamous cell carcinoma. Cancer. Published online 11 December 2019. http s://doi.org/10.1002/cncr.32654.\%3caq3>

14. Cracchiolo JR, Baxi SS, Morris LG, et al. Increase in primary surgical treatment of $\mathrm{T} 1$ and $\mathrm{T} 2$ oropharyngeal squamous cell carcinoma and rates of adverse pathologic features: National Cancer Data Base. Cancer. 2016;122:1523-32. https://doi.org/10. 1002/cncr.29938.

15. SEER Program Code Manual 3rd ed. rev 1. SEER Field and Code Changes for 2003. Published online 2003:26.

16. Rosenthal DI, Liu L, Lee JH, et al. Importance of the treatment package time in surgery and postoperative radiation therapy for squamous carcinoma of the head and neck. Head Neck. 2002;24:115-26. https://doi.org/10.1002/hed.10038.

17. Eskander A, Irish J, Groome PA, et al. Volume-outcome relationships for head and neck cancer surgery in a universal health care system. Laryngoscope. 2014;124:2081-8. https://doi.org/10. 1002/lary.24704.

18. Birkmeyer JD, Siewers AE, Finlayson EVA, et al. Hospital volume and surgical mortality in the United States. $N$ Engl J Med. 2002;346:1128-37. https://doi.org/10.1056/nejmsa012337.

19. Wuthrick EJ, Zhang Q, Machtay M, et al. Institutional clinical trial accrual volume and survival of patients with head and neck cancer. JCO. 2014;33:156-64. https://doi.org/10.1200/jco.2014. 56.5218 .

20. Hollenbeck BK, Dunn RL, Miller DC, Daignault S, Taub DA, Wei JT. Volume-based referral for cancer surgery: informing the debate. J Clin Oncol. 2007;25:91-6. https://doi.org/10.1200/jco. 2006.07.2454.

21. Finlayson EVA, Goodney PP, Birkmeyer JD. Hospital volume and operative mortality in cancer surgery: a national study. Arch Surg. 2003;138:721-725. discussion 726. https://doi.org/10.1001 /archsurg.138.7.721

22. McCahill LE, Privette A, James T, et al. Quality measures for breast cancer surgery: initial validation of feasibility and assessment of variation among surgeons. Arch Surg. 2009;144:455-62; discussion 462-3. https://doi.org/10.1001/arch surg. 2009.56

Publisher's Note Springer Nature remains neutral with regard to jurisdictional claims in published maps and institutional affiliations. 